\title{
TRANSFORMATION OF THE LINEAR DIFFERENCE EQUATION INTO A SYSTEM OF THE FIRST ORDER DIFFERENCE EQUATIONS
}

\author{
M.I. Ayzatsky \\ National Science Center “Kharkov Institute of Physics and Technology”, Kharkiv, Ukraine \\ E-mail: aizatsky@kipt.kharkov.ua
}

The transformation of the $N$-th-order linear difference equation into a system of the first order difference equations is presented. The proposed transformation opens possibility to obtain new forms of the $N$-dimensional system of the first order equations that can be useful for the analysis of solutions of the $N$-th-order difference equations. In particular for the third-order linear difference equation the nonlinear second-order difference equation that plays the same role as the Riccati equation for second-order linear difference equation is obtained. The new form of the $N$ dimensional system of first order equations can also be used to find the WKB solutions of the linear difference equation with coefficients that vary slowly with index

PACS: $02.30 . \mathrm{Hq}$

\section{INTRODUCTION}

It is common knowledge that a difference equation of order $N$

$$
\begin{aligned}
& y_{k+N}+f_{N-1, k} y_{k+N-1}+f_{N-2, k} y_{k+N-2}+\ldots \\
& +f_{2, k} y_{k+2}+f_{1, k} y_{k+1}+f_{0, k} y_{k}+f_{k}=0
\end{aligned}
$$

may be transformed in a standard way to a system of the $N$ first-order difference equations. To obtain such transformation we introduce a number of new variables (see, for example, $[1,2]$ )

$$
x_{k}^{(i)}=y_{k+i-1}, i=1,2, \ldots, N .
$$

The difference equation (1) can be rewritten as

$$
X_{k+1}=T_{k} X_{k}+F_{k},
$$

where $\quad X_{k}=\left(x_{k+N-1}, x_{k+N-1}, \ldots, x_{k}\right)^{T}, F_{k}=\left(f_{k}, 0, \ldots, 0\right)^{T}$, and the companion matrix of (1) is

$$
T_{k}=\left(\begin{array}{ccccc}
-f_{N-1, k} & -f_{N-2, k} & \ldots & -f_{1, k} & -f_{0, k} \\
1 & 0 & \ldots & 0 & 0 \\
0 & 1 & \ldots & 0 & 0 \\
0 & 0 & \ldots & 1 & 0
\end{array}\right) .
$$

In fact we do not introduce new variables ${ }^{1}$, we only do re-designations and still work with the elements of the same sequence $y_{k}$.

There is another kind of transformation ${ }^{2}$ that consists of representation of the solution $y_{k}$ of the equation (1) as the sum of the $N$ new unknown grid functions [3 -5]. By introducing $N$ new unknowns, instead of the one, we can impose $(N-1)$ additional conditions. Such approach gives new form of the $N$-dimensional system of first order equations, equivalent to the equation (1). In this article some generalization of the proposed transformation [3] is given. Analysis of literature shows that it apparently has not been described earlier.

\footnotetext{
${ }^{1}$ In the case of transformation of differential equations we do introduce new variables $x^{(i)}=d^{i-1} y / d t^{i-1}, i=1,2, \ldots, N$.

${ }^{2}$ For the case of second-order differential equations it was used in [6].
}

\section{TRANSFORMATION THE $N$-th-ORDER LINEAR DIFFERENCE EQUATION}

We represent the solution of the difference equation (1) as the sum of new grid functions

$$
y_{k}=\sum_{n=1}^{N} y_{n, k} \text {. }
$$

By introducing $N$ new unknowns $y_{n, k}$ instead of the one $y_{k}$, we can impose additional conditions. These conditions we write in the form

$$
\begin{aligned}
& y_{k+1}=\sum_{n=1}^{N} g_{1, n, k} y_{n, k}, \\
& y_{k+2}=\sum_{n=1}^{N} g_{2, n, k} y_{n, k}, \\
& \ldots \\
& y_{k+N-1}=\sum_{n=1}^{N} g_{N-1, n, k} y_{n, k},
\end{aligned}
$$

where $g_{m, n, k}(1 \leq n \leq N, 1 \leq m \leq N-1)$ are the arbitrary sequences.

If

$$
\operatorname{det}\left(\begin{array}{cccc}
1 & 1 & . . & 1 \\
g_{1,1, k} & g_{1,2, k} & \cdots & g_{1, N, k} \\
\cdots & \cdots & \cdots & \cdots \\
g_{N-1,1, k} & g_{N-1,2, k} & \cdots & g_{N-1, N, k}
\end{array}\right) \neq 0
$$

then the representation (5), (6) is unique. Indeed, from (5) and (6) we can uniquely find $y_{n, k}$ as a linear combination of $y_{k}$. Using (1), (5), and (6) we can write such system of equations

$$
\begin{gathered}
y_{k+1}=\sum_{n=1}^{N} y_{n, k+1}=\sum_{n=1}^{N} g_{1, n, k} y_{n, k}, \\
y_{k+2}=\sum_{n=1}^{N} g_{1, n, k+1} y_{n, k+1}=\sum_{n=1}^{N} g_{2, n, k} y_{n, k}, \\
\ldots \\
y_{k+N-1}=\sum_{n=1}^{N} g_{N-2, n, k+1} y_{n, k+1}=\sum_{n=1}^{N} g_{N-1, n, k} y_{n, k}, \\
\sum_{n=1}^{N} g_{N-1, n, k+1} y_{n, k+1}= \\
=-\sum_{n=1}^{N}\left(\sum_{m=1}^{N-1} f_{N-m, k} g_{N-m, n, k}+f_{0, k}\right) y_{n, k}-f_{k} .
\end{gathered}
$$


In matrix form

$$
M_{k+1} Y_{k+1}=H_{k+1} Y_{k}+F_{k+1},
$$

where $Y_{k}=\left(y_{1, k}, y_{2, k}, \ldots, y_{N, k}\right)^{T}, F_{k}=\left(0,0 \ldots,-f_{k}\right)^{T}$,

$$
\begin{gathered}
M_{k}=\left(\begin{array}{cccc}
1 & 1 & . . & 1 \\
g_{1,1, k} & g_{1,2, k} & \ldots & g_{1, N, k} \\
\ldots & \ldots & \ldots & \ldots \\
g_{N-1,1, k} & g_{N-1,2, k} & \ldots & g_{N-1, N, k}
\end{array}\right), \\
H_{k+1}=\left(\begin{array}{cccc}
g_{1,1, k} & g_{1,2, k} & \ldots & g_{1, N, k} \\
\ldots & \ldots & \ldots & \ldots \\
g_{N-1,1, k} & g_{N-1,2, k} & \ldots & g_{N-1, N, k} \\
A_{1, k-1} & A_{2, k-1} & \ldots & A_{N, k-1}
\end{array}\right), \\
A_{n, k}=-\left(\sum_{m=1}^{N-1} f_{N-m, k} g_{N-m, n, k}+f_{0, k}\right) .
\end{gathered}
$$

And finally, we have the equation

$$
Y_{k+1}=T_{k+1} Y_{k}+\bar{F}_{k+1},
$$

where $T_{k}=M_{k}^{-1} H_{k}, \bar{F}_{k}=M_{k}^{-1} F_{k}$.

We would like to emphasize that the sequences $g_{m, n, k}$ are the arbitrary ones, and we do not impose a condition that the new grid functions $y_{m, k}$ are the solutions of the equation (1).

As $g_{m, n, k}$ are the arbitrary sequences we can try to find such sequences that result in diagonal matrix $T_{k}$. If it can be done, we easily find the solution of the system (12) and the initial difference equation (1). It can be shown that in this case $y_{m, k}$ are the linearly independent solutions of the equation (1).

\section{TRANSFORMATION THE SECOND- ORDER LINEAR DIFFERENCE EQUATION}

Following the section 2 we represent the solution of the linear second-order equation

$$
y_{k+2}+f_{1, k} y_{k+1}+f_{0, k} y_{k}+f_{k}=0
$$

as the sum of the two new grid functions

$$
y_{k}=y_{1, k}+y_{2, k} \text {. }
$$

We write an additional condition as

$$
y_{k+1}=g_{1, k} y_{1, k}+g_{2, k} y_{2, k},
$$

where $g_{n, k}(1 \leq n \leq 2)$ are the arbitrary sequences.

Applying transformations from section 2, we can write such system of equations

$$
\left(\begin{array}{l}
y_{1, k+1} \\
y_{2, k+1}
\end{array}\right)=T_{k+1}\left(\begin{array}{l}
y_{1, k} \\
y_{2, k}
\end{array}\right)+F_{k+1},
$$

where

$$
\begin{gathered}
T_{k+1}=\left(\begin{array}{cc}
-\frac{f_{0, k}+g_{1, k}\left(g_{2, k+1}+f_{1, k}\right)}{g_{1,1, k+1}-g_{1,2, k+1}} & -\frac{f_{0, k}+g_{2, k}\left(g_{2, k+1}+f_{1, k}\right)}{g_{1, k+1}-g_{2, k+1}} \\
\frac{f_{0, k}+g_{1, k}\left(g_{1, k+1}+f_{1, k}\right)}{g_{1, k+1}-g_{2, k+1}} & \frac{f_{0, k}+g_{2, k}\left(g_{1, k+1}+f_{1, k}\right)}{g_{1, k+1}-g_{2, k+1}}
\end{array}\right), \\
F_{k+1}=\left(\begin{array}{l}
-\frac{f_{k+1}}{g_{1, k+1}-g_{2, k+1}} \\
\frac{f_{k+1}}{g_{1, k+1}-g_{2, k+1}}
\end{array}\right)=\left(\begin{array}{l}
-\bar{f}_{k+1} \\
\bar{f}_{k+1}
\end{array}\right) .
\end{gathered}
$$

We will consider the homogeneous difference equations $\left(f_{k}=0\right)$. The normal system of difference equations (16) can be transformed into the known ones.

From (17) it follows that we can choose the sequences $g_{(1,2), k}$ in such a way that matrix $T_{k}$ will be triangular or even diagonal one. It is realized by setting $T_{k, 12}=0$ and $T_{k, 21}=0$.

These conditions give the non-linear second-order rational difference equation (Riccaty type [3, 7 - 9]) for the sequences $g_{(1,2), k}$

$$
f_{0, k}+g_{(1,2), k}\left(g_{(1,2), k+1}+f_{1, k}\right)=0 \text {. }
$$

In this case the matrix $T_{k}$ is a diagonal one and the system (16) takes the form:

$$
y_{(1,2), k+1}=g_{(1,2), k} y_{(1,2), k} .
$$

Solutions $y_{1, k}, y_{2, k}$ are linearly independent.

The characteristic equation of the difference equation (13) is

$$
\rho_{k}^{2}+f_{1, k} \rho_{k}+f_{0, k}=0 .
$$

Let $g_{(1,2), k}=\rho_{k}^{(1,2)}$, where $\rho_{k}^{(1,2)}$ are the solutions of the characteristic equation (21)

$$
\rho_{k}^{(1,2)}=-\frac{f_{1, k}}{2} \pm \frac{1}{2} \sqrt{f_{1, k}^{2}-4 f_{0 . k}} .
$$

The matrix $T_{k}$ takes the form

$$
T_{k+1}=\left(\begin{array}{ccc}
\rho_{k}^{(1)} \frac{\rho_{k}^{(1)}-\rho_{k+1}^{(2)}}{\rho_{k+1}^{(1)}-\rho_{k+1}^{(2)}} & \rho_{k}^{(2)} \frac{\rho_{k}^{(2)}-\rho_{k+1}^{(2)}}{\rho_{k+1}^{(1)}-\rho_{k+1}^{(2)}} \\
\rho_{k}^{(1)} \frac{\rho_{k+1}^{(1)}-\rho_{k}^{(1)}}{\rho_{k+1}^{(1)}-\rho_{k+1}^{(2)}} & \rho_{k}^{(2)} \frac{\rho_{k+1}^{(1)}-\rho_{k}^{(2)}}{\rho_{k+1}^{(1)}-\rho_{k+1}^{(2)}}
\end{array}\right) .
$$

If sequences $f_{(0,1), k}$ vary sufficiently slowly with $k$ $\left(f_{(0,1), k}=f_{(0,1)}(\varepsilon k), \quad 0 \leq \varepsilon \ll 1\right)$, then the differences $\left(\rho_{k+1}^{(1,2)}-\rho_{k}^{(1,2)}\right)$ are small and we can neglect the nondiagonal terms in the matrix $T_{k}$. This gives

$$
\begin{aligned}
& y_{1, k+1}=\rho_{k}^{(1)}\left(1-\frac{\rho_{k+1}^{(1)}-\rho_{k}^{(1)}}{\rho_{k+1}^{(1)}-\rho_{k+1}^{(2)}}\right) y_{1, k}, \\
& y_{2, k+1}=\rho_{k}^{(2)}\left(1+\frac{\rho_{k+1}^{(2)}-\rho_{k}^{(2)}}{\rho_{k+1}^{(1)}-\rho_{k+1}^{(2)}}\right) y_{2, k} .
\end{aligned}
$$

It can be shown that these equations coincide with the equations of the discrete WKB approach (see, for example, $[10,11])$. Indeed, from (20) it follows that the discrete WKB equations can be obtained by using an approximate solutions of the Riccati equation (19) under assumption that $f_{0, k}$ and $f_{1, k}$ vary sufficiently slowly with $k$. The Riccati equations can be transformed by an iteration procedure into the quadratic equations

$$
\left(g_{(1,2), k}\right)^{2}+g_{(1,2), k} f_{1, k}+f_{0, k}+\rho_{k+1}^{(1,2)}\left(\rho_{k+2}^{(1,2)}-\rho_{k+1}^{(1,2)}\right)=0 .
$$

It is one of the possible forms of the quadratic equation (compare with $[10,11]$ ) that can be obtained at the second iteration. Its solutions differ from the ones that were obtained in $[10,11]$ by an amount of order $\varepsilon^{2}$. The approximate solutions of this equations with error of $O\left(\varepsilon^{2}\right)$ are 


$$
g_{(1,2), k} \approx \rho_{k}^{(1,2)}\left(1 \mp \frac{\rho_{k+1}^{(1,2)}-\rho_{k}^{(1,2)}}{\rho_{k+1}^{(1)}-\rho_{k+1}^{(2)}}\right) .
$$

Comparison (24) and (26) shows that these two different approaches give the same result, and the equations (24) and (20) coincide.

The solutions of the equations (24) at $k>k_{0}$ can be written as

$$
\begin{gathered}
y_{k}^{(1,2)}=\prod_{s=k_{0}+1}^{k} T_{s, 11,22} y_{k_{0}}^{(1)}=y_{k_{0}}^{(1,2)} \exp \left(\sum_{s=k_{0}+1}^{k} \ln \rho_{s-1}^{(1,2)}-\right. \\
\left.-\sum_{s=k_{0}+1}^{k} \frac{\sqrt{f_{1, s}^{2}-4 f_{0, s}}-\sqrt{f_{1, s-1}^{2}-4 f_{0, s-1}}}{2 \sqrt{f_{1, s}^{2}-4 f_{0, s}}} \pm \sum_{s=k_{0}+1}^{k} \frac{f_{1, s}-f_{1, s-1}}{2 \sqrt{f_{1, s}^{2}-4 f_{0, s}}}\right) \sim \\
\sim \frac{1}{\left(f_{1, k}^{2}-4 f_{0 . k}\right)^{1 / 4}} \exp \left(\sum_{s=k_{0}+1}^{k} \ln \rho_{s-1}^{(1,2)} \pm \sum_{s=k_{0}+1}^{k} \frac{f_{1, s}-f_{1, s-1}}{2 \sqrt{f_{1, s}^{2}-4 f_{0, s}}}\right) .
\end{gathered}
$$

Comparison of this formula with that obtained by directly finding an approximate solution from the equation (1) [12] gives some difference. The formula (27) contains additional sum in the exponent (the second sum).

\section{TRANSFORMATION THE THIRD-ORDER LINEAR DIFFERENCE EQUATION}

Let's represent the solution of the linear third-order equation

$$
y_{k+3}+f_{2, k} y_{k+2}+f_{1, k} y_{k+1}+f_{0, k} y_{k}+f_{k}=0
$$

as the sum of the three new functions

$$
y_{k}=y_{1, k}+y_{2, k}+y_{3, k} \text {. }
$$

We write additional conditions in the form

$$
\begin{aligned}
& y_{k+1}=g_{1,1, k} y_{1, k}+g_{1,2, k} y_{2, k}+g_{1,3, k} y_{3, k}, \\
& y_{k+2}=g_{2,1, k} y_{1, k}+g_{2,2, k} y_{2, k}+g_{2,3, k} y_{3, k} .
\end{aligned}
$$

Applying the transformations that are given in section 2 , we obtain a system of the first-order linear difference equations

$$
\begin{aligned}
& y_{1, k+1} D_{k+1}=g_{1,1, k} y_{1, k} D_{k+1}+ \\
& +y_{1, k}\left[\begin{array}{c}
g_{1,1, k}\left\{\begin{array}{l}
\left(g_{1,1, k+1}-g_{1,1, k}\right)\left(g_{2,3, k+1}-g_{2,2, k+1}\right)+ \\
+\left(g_{2,1, k+1}-g_{2,1, k}\right)\left(g_{1,2, k+1}-g_{1,3, k+1}\right)
\end{array}\right\}+ \\
+x_{1, k}\left(g_{2,3, k+1}-g_{2,2, k+1}\right)+\left(g_{1,2, k+1}-g_{1,3, k+1}\right) x_{4, k}
\end{array}\right]+ \\
& +y_{2, k}\left[\begin{array}{c}
g_{1,2, k}\left\{\begin{array}{l}
\left(g_{1,2, k+1}-g_{1,2, k}\right)\left(g_{2,3, k+1}-g_{2,2, k+1}\right)+ \\
+\left(g_{2,2, k+1}-g_{2,2, k}\right)\left(g_{1,2, k+1}-g_{1,3, k+1}\right)
\end{array}\right\}+ \\
+x_{2, k}\left(g_{2,3, k+1}-g_{2,2, k+1}\right)+\left(g_{1,2, k+1}-g_{1,3, k+1}\right) x_{5, k}
\end{array}\right] \\
& +y_{3, k}\left[\begin{array}{c}
g_{1,3, k}\left\{\begin{array}{l}
\left(g_{1,3, k+1}-g_{1,3, k}\right)\left(g_{2,3, k+1}-g_{2,2, k+1}\right)+ \\
+\left(g_{2,3, k+1}-g_{2,3, k}\right)\left(g_{1,2, k+1}-g_{1,3, k+1}\right)
\end{array}\right\}^{\prime}+ \\
+x_{3, k}\left(g_{2,3, k+1}-g_{2,2, k+1}\right)+\left(g_{1,2, k+1}-g_{1,3, k+1}\right) x_{6, k}
\end{array}\right] \\
& -f_{k}\left(g_{1,3, k+1}-g_{1,2, k+1}\right) \text {, } \\
& y_{2, k+1} D_{k+1}=y_{2, k} g_{1,2, k} D_{k+1}+ \\
& +y_{1, k}\left[\begin{array}{c}
g_{1,1, k}\left\{\begin{array}{l}
\left(g_{1,1, k+1}-g_{1,1, k}\right)\left(g_{2,1, k+1}-g_{2,3, k+1}\right)+ \\
+\left(g_{2,1, k+1}-g_{2,1, k}\right)\left(g_{1,3, k+1}-g_{1,1, k+1}\right)
\end{array}\right\}+ \\
+x_{1, k}\left(g_{2,1, k+1}-g_{2,3, k+1}\right)+\left(g_{1,3, k+1}-g_{1,1, k+1}\right) x_{4, k}
\end{array}\right]+ \\
& +y_{2, k}\left[\begin{array}{c}
g_{1,2, k}\left\{\begin{array}{l}
\left(g_{1,2, k+1}-g_{1,2, k}\right)\left(g_{2,1, k+1}-g_{2,3, k+1}\right)+ \\
+\left(g_{2,2, k+1}-g_{2,2, k}\right)\left(g_{1,3, k+1}-g_{1,1, k+1}\right)
\end{array}\right\}+ \\
+x_{2, k}\left(g_{2,1, k+1}-g_{2,3, k+1}\right)+\left(g_{1,3, k+1}-g_{1,1, k+1}\right) x_{5, k}
\end{array}\right] \\
& +y_{3, k}\left[\begin{array}{c}
g_{1,3, k}\left\{\begin{array}{l}
\left(g_{1,3, k+1}-g_{1,3, k}\right)\left(g_{2,1, k+1}-g_{2,3, k+1}\right)+ \\
+\left(g_{2,3, k+1}-g_{2,3, k}\right)\left(g_{1,3, k+1}-g_{1,1, k+k+1}\right)
\end{array}\right)+ \\
+x_{3, k}\left(g_{2,1, k+1}-g_{2,3, k+1}\right)+\left(g_{1,3, k+1}-g_{1,1, k+1}\right) x_{6, k}
\end{array}\right]+ \\
& -f_{k}\left(g_{1,1, k+1}-g_{1,3, k+1}\right) \text {, }
\end{aligned}
$$

$$
\begin{aligned}
& y_{3, k+1} D_{k+1}=y_{3, k} g_{1,3, k} D_{k+1}+ \\
& +y_{1, k}\left[\begin{array}{l}
g_{1,1, k}\left\{\begin{array}{l}
\left(g_{1,1, k+1}-g_{1,1, k}\right)\left(g_{2,2, k+1}-g_{2,1, k+1}\right)+ \\
+\left(g_{2,1, k+1}-g_{2,1, k}\right)\left(g_{1,1, k+1}-g_{1,2, k+1}\right)
\end{array}\right\} \\
+x_{1, k}\left(g_{2,2, k+1}-g_{2,1, k+1}\right)+\left(g_{1,1, k+1}-g_{1,2, k+1}\right) x_{4, k}
\end{array}\right]+ \\
& +y_{2, k}\left[\begin{array}{l}
g_{1,2, k}\left\{\begin{array}{l}
\left(g_{1,2, k+1}-g_{1,2, k}\right)\left(g_{2,2, k+1}-g_{2,1, k+1}\right)+ \\
+\left(g_{2,2, k+1}-g_{2,2, k}\right)\left(g_{1,1, k+1}-g_{1,2, k+1}\right)+
\end{array}\right. \\
+x_{2, k}\left(g_{2,2, k+1}-g_{2,1, k+k+1}\right)+\left(g_{1,1, k+1}-g_{1,2, k+1}\right) x_{5, k}
\end{array}\right\} \\
& +y_{3, k}\left[\begin{array}{l}
g_{1,3, k}\left\{\begin{array}{l}
\left(g_{1,3, k+1}-g_{1,3, k}\right)\left(g_{2,2, k+1}-g_{2,1, k+1}\right)+ \\
+\left(g_{2,3, k+1}-g_{2,3, k}\right)\left(g_{1,1, k+1}-g_{1,2, k+1}\right)
\end{array}\right\}+ \\
+x_{3, k}\left(g_{2,2, k+1}-g_{2,1, k+1}\right)+\left(g_{1,1, k+1}-g_{1,2, k+1}\right) x_{6, k}
\end{array}\right] \\
& -f_{k}\left(g_{1,2, k+1}-g_{1,1, k+1}\right) .
\end{aligned}
$$

where the following notations were introduced

$$
\begin{aligned}
& x_{1, k}=\left(g_{1,1, k}\right)^{2}-g_{2,1, k}, \\
& x_{2, k}=\left(g_{1,2, k}\right)^{2}-g_{2,2, k}, \\
& x_{3, k}=\left(g_{1,3, k}\right)^{2}-g_{2,3, k}, \\
& x_{4, k}=g_{1,1, k} g_{2,1, k}+f_{2, k} g_{2,1, k}+f_{1, k} g_{1,1, k}+f_{0, k}, \\
& x_{5, k}=g_{1,2, k} g_{2,2, k}+f_{2, k} g_{2,2, k}+f_{1, k} g_{1,2, k}+f_{0, k}, \\
& x_{6, k}=g_{1,3, k} g_{2,3, k}+f_{2, k} g_{2,3, k}+f_{1, k} g_{1,3, k}+f_{0, k} .
\end{aligned}
$$

If we choose

$$
g_{1, n, k}=\rho_{k}^{(n)}, g_{2, n, k}=\rho_{k}^{(n) 2}, n=1,2,3,
$$

where $\rho_{k}^{(n)}$ are the solutions of the equation

$$
\rho_{k}^{3}+f_{2, k} \rho_{k}^{2}+f_{1, k} \rho_{k}+f_{0, k}=0,
$$

then $x_{i, k}=0, i=1, \ldots, 6$ and the system (31) - (33) takes the form

$$
\begin{aligned}
& y_{1, k+1}=y_{1, k} \rho_{k}^{(1)}+ \\
& y_{1, k} \rho_{k}^{(1)}\left(\rho_{k+1}^{(1)}-\rho_{k}^{(1)}\right)\left(\rho_{k+1}^{(3)}-\rho_{k+1}^{(2)}\right)\left[\left(\rho_{k+1}^{(3)}+\rho_{k+1}^{(2)}\right)-\left(\rho_{k+1}^{(1)}+\rho_{k}^{(1)}\right)\right] / D_{k+1}+ \\
& y_{2, k} \rho_{k}^{(2)}\left(\rho_{k+1}^{(2)}-\rho_{k}^{2)}\right)\left(\rho_{k+1}^{(3)}-\rho_{k+1}^{(2)}\right)\left[\left(\rho_{k+1}^{(3)}+\rho_{k+1}^{(2)}\right)-\left(\rho_{k+1}^{(2)}+\rho_{k}^{(2)}\right)\right] / D_{k+1}(37) \\
& y_{3, k} \rho_{k}^{(3)}\left(\rho_{k+1}^{(3)}-\rho_{k}^{(3)}\right)\left(\rho_{k+1}^{(3)}-\rho_{k+1}^{(2)}\right)\left[\left(\rho_{k+1}^{(3)}+\rho_{k+1}^{(2)}\right)-\left(\rho_{k+1}^{(3)}+\rho_{k}^{(3)}\right)\right] / D_{k+1} \\
& -f_{k}\left(\rho_{k+1}^{(3)}-\rho_{k+1}^{(2)}\right) / D_{k+1}, \\
& y_{2, k+1}=y_{2, k} \rho_{k}^{(2)}+ \\
& y_{1, k} \rho_{k}^{(1)}\left(\rho_{k+1}^{(1)}-\rho_{k}^{(1)}\right)\left(\rho_{k+1}^{(1)}-\rho_{k+1}^{(3)}\right)\left[\left(\rho_{k+1}^{(1)}+\rho_{k+1}^{(3)}\right)-\left(\rho_{k+1}^{(1)}+\rho_{k}^{(1)}\right)\right] / D_{k+1}+ \\
& y_{2, k} \rho_{k}^{(2)}\left(\rho_{k+1}^{(2)}-\rho_{k}^{(2)}\right)\left(\rho_{k+1}^{(1)}-\rho_{k+1}^{(3)}\right)\left[\left(\rho_{k+1}^{(1)}+\rho_{k+1}^{(3)}\right)-\left(\rho_{k+1}^{(2)}+\rho_{k}^{(2)}\right)\right] / D_{k+1}+(38) \\
& y_{3, k} \rho_{k}^{(3)}\left(\rho_{k+1}^{(3)}-\rho_{k}^{(3)}\right)\left(\rho_{k+1}^{(1)}-\rho_{k+1}^{(3)}\right)\left[\left(\rho_{k+1}^{(1)}+\rho_{k+1}^{(3)}\right)-\left(\rho_{k+1}^{(3)}+\rho_{k}^{(3)}\right)\right] / D_{k+1}- \\
& -f_{k}\left(\rho_{k+1}^{(1)}-\rho_{k+1}^{(3)}\right) / D_{k+1}, \\
& y_{3, k+1}=y_{3, k} \rho_{k}^{(3)}+ \\
& y_{1, k} \rho_{k}^{(1)}\left(\rho_{k+1}^{(1)}-\rho_{k}^{(1)}\right)\left(\rho_{k+1}^{(2)}-\rho_{k+1}^{(1)}\right)\left[\left(\rho_{k+1}^{(2)}+\rho_{k+1}^{(1)}\right)-\left(\rho_{k+1}^{(1)}+\rho_{k}^{(1)}\right)\right] / D_{k+1}+ \\
& y_{2, k} \rho_{k}^{(2)}\left(\rho_{k+1}^{(2)}-\rho_{k}^{(2)}\right)\left(\rho_{k+1}^{(2)}-\rho_{k+1}^{(1)}\right)\left[\left(\rho_{k+1}^{(2)}+\rho_{k+1}^{(1)}\right)-\left(\rho_{k+1}^{(2)}+\rho_{k}^{(2)}\right)\right] / D_{k+1}+(39) \\
& y_{3, k} \rho_{k}^{(3)}\left(\rho_{k+1}^{(3)}-\rho_{k+1}^{(3)}\right)\left(\rho_{k+1}^{(2)}-\rho_{k+1}^{(1)}\right)\left[\left(\rho_{k+1}^{(2)}+\rho_{k+1}^{(1)}\right)-\left(\rho_{k+1}^{(3)}+\rho_{k}^{(3)}\right)\right] / D_{k+1}- \\
& -f_{k}\left(\rho_{k+1}^{(2)}-\rho_{k+1}^{(1)}\right) / D_{k+1},
\end{aligned}
$$

where $\quad D_{k+1}=\left(\rho_{k+1}^{(2)}-\rho_{k+1}^{(1)}\right)\left(\rho_{k+1}^{(3)}-\rho_{k+1}^{(1)}\right)\left(\rho_{k+1}^{(3)}-\rho_{k+1}^{(2)}\right)-$ the Vandermonde determinant.

If the sequences $f_{0, k}, f_{1, k}, f_{2, k}$ vary sufficiently slowly with $k \quad\left(f_{0, k}=f_{0}(\varepsilon k), \quad f_{1, k}=f_{1}(\varepsilon k)\right.$, $\left.f_{2, k}=f_{2}(\varepsilon k), \quad 0 \leq \varepsilon \ll 1\right)$, then the differences $\left(\rho_{k+1}^{(n)}-\rho_{k}^{(n)}\right)$ are the small values and we can neglect the non-diagonal terms in the matrix $T_{k}$. This gives the WKB approximation 


$$
\begin{gathered}
y_{1, k+1} \approx y_{1, k} \rho_{k}^{(1)}- \\
-y_{1, k} \rho_{k}^{(1)}\left(\rho_{k+1}^{(1)}-\rho_{k}^{(1)}\right)\left[\frac{1}{\left(\rho_{k+1}^{(1)}-\rho_{k+1}^{(2)}\right)}+\frac{1}{\left(\rho_{k+1}^{(1)}-\rho_{k+1}^{(3)}\right)}\right]-f_{k} \frac{\left(\rho_{k+1}^{(3)}-\rho_{k+1}^{(2)}\right)}{D_{k+1}}, \\
y_{2, k+1} \approx y_{2, k} \rho_{k}^{(2)}- \\
-y_{2, k} \rho_{k}^{(2)}\left(\rho_{k+1}^{(2)}-\rho_{k}^{(2)}\right)\left[\frac{1}{\left(\rho_{k+1}^{(2)}-\rho_{k+1}^{(3)}\right)}+\frac{1}{\left(\rho_{k+1}^{(2)}-\rho_{k+1}^{(1)}\right)}\right]-f_{k} \frac{\left(\rho_{k+1}^{(1)}-\rho_{k+1}^{(3)}\right)}{D_{k+1}}, \\
y_{3, k+1} \approx y_{3, k} \rho_{k}^{(3)}- \\
-y_{3, k} \rho_{k}^{(3)}\left(\rho_{k+1}^{(3)}-\rho_{k}^{(3)}\right)\left[\frac{1}{\left(\rho_{k+1}^{(3)}-\rho_{k+1}^{(1)}\right)}+\frac{1}{\left(\rho_{k+1}^{(3)}-\rho_{k+1}^{(2)}\right)}\right]-f_{k} \frac{\left(\rho_{k+1}^{(2)}-\rho_{k+1}^{(1)}\right)}{D_{k+1}} .
\end{gathered}
$$

If we choose the sequences $g_{m . n, k}$ to be the solutions of the following equations

$$
\begin{aligned}
& x_{1, k}=\left(g_{1,1, k}\right)^{2}-g_{2,1, k}=-g_{1,1, k}\left(g_{1,1, k+1}-g_{1,1, k}\right), \\
& x_{4, k}=g_{1,1, k} g_{2,1, k}+f_{2, k} g_{2,1, k}+f_{1, k} g_{1,1, k}+f_{0, k}=-g_{1,1, k}\left(g_{2,1, k+1}-g_{2,1, k}\right), \\
& x_{2, k}=\left(g_{1,2, k}\right)^{2}-g_{2,2, k}=-g_{1,2, k}\left(g_{1,2, k+1}-g_{1,2, k}\right), \\
& x_{5, k}=g_{1,2, k} g_{2,2, k}+f_{2, k} g_{2,2, k}+f_{1, k} g_{1,2, k}+f_{0, k}=-g_{1,2, k}\left(g_{2,2, k+1}-g_{2,2, k}\right), \\
& x_{3, k}=\left(g_{1,3, k}\right)^{2}-g_{2,3, k}=-g_{1,3, k}\left(g_{1,3, k+1}-g_{1,3, k}\right), \\
& x_{6, k}=g_{1,3, k} g_{2,3, k}+f_{2, k} g_{2,3, k}+f_{1, k} g_{1,3, k}+f_{0, k}=-g_{1,3, k}\left(g_{2,3, k+1}-g_{2,3, k}\right),
\end{aligned}
$$

the system (33) takes form

$$
\begin{aligned}
& y_{1, k+1}=y_{1, k} g_{1,1, k}-f_{k} \frac{\left(g_{1,3, k+1}-g_{1,2, k+1}\right)}{D_{k+1}}, \\
& y_{2, k+1}=y_{2, k} g_{1,2, k}-f_{k} \frac{\left(g_{1,1, k+1}-g_{1,3, k+1}\right)}{D_{k+1}}, \\
& y_{3, k+1}=y_{3, k} g_{1,3, k}-f_{k} \frac{\left(g_{1,2, k+1}-g_{1,1, k+1}\right)}{D_{k+1}} .
\end{aligned}
$$

From (43) it follows that sequences $g_{m . n, k}$ are the three different solutions of the system of the first-order nonlinear difference equations

$$
\begin{aligned}
& p_{k}^{(1)}\left(p_{k+1}^{(1)}-p_{k}^{(1)}\right)+p_{k}^{(1) 2}-p_{k}^{(2)}=0, \\
& p_{k}^{(1)}\left(p_{k+1}^{(2)}-p_{k}^{(2)}\right)+f_{2, k} p_{k}^{(2)}+f_{1, k} p_{k}^{(1)}+f_{0, k}+p_{k}^{(2)} p_{k}^{(1)}=0 .
\end{aligned}
$$

This system can be written as the second-order nonlinear difference equation

$$
p_{k+2}^{(1)} p_{k+1}^{(1)} p_{k}^{(1)}+f_{2, k} p_{k+1}^{(1)} p_{k}^{(1)}+f_{1, k} p_{k}^{(1)}+f_{0, k}=0 .
$$

For the third-order linear difference equation (28) the equation (48) (or system (47)) plays the same role as the Riccati equation for second-order linear difference equation.

The functions $y_{n, k}=\prod_{k} g_{1, n, k}$ are linear independent, and the general solution of the homogeneous equation (28) $\left(f_{k}=0\right)$ is

$$
y_{k}=\sum_{n=1}^{3} y_{n, k_{0}} \prod_{s=k_{0}}^{k-1} g_{1, n, s} .
$$

There are other forms of the system of the first order equations that can be obtained from the system (33) by choosing different sequences $g_{m, n, k}$.

Finding the WKB solutions of the linear difference equation (28) with coefficients that vary sufficiently slowly with index $k$ by finding the three iteration solutions of the equation (48) is not a simple procedure (compare with $[10,11]$ ). So it seems preferable to use the approach that leads us to the WKB equations (40)(42).

\section{THE WKB APPROXIMATION \\ FOR THE $N$-th-ORDER LINEAR DIFFERENCE EQUATION}

Sections' 3 and 4 results show that WKB equations for the $\mathrm{N}$-order linear difference equation with coefficients that vary sufficiently slowly with index can be obtained by choosing sequences $g_{m, n, k}=\left(\rho_{k}^{(n)}\right)^{m}$, where $\rho_{k}^{(n)}$ are the solutions of the characteristic equation

$$
\begin{aligned}
& \rho_{k}^{N}+f_{N-1, k} \rho_{k}^{N-1}+f_{N-2, k} \rho_{k}^{N-2}+\ldots \\
& +f_{2, k} \rho_{k}^{2} y_{k+2}+f_{1, k} \rho_{k}+f_{0, k}=0
\end{aligned}
$$

and taking into consideration only diagonal elements of the matrix $T_{k}$ in the equation

$$
Y_{k+1}=T_{k+1} Y_{k}=M_{k+1}^{-1} H_{k+1} Y_{k} .
$$

If we chose $g_{m, n, k}=\left(\rho_{k}^{(n)}\right)^{m}$, the matrix $M_{k}$ transforms into the Vandermonde matrix and we can find its inverse [13]

$$
M_{k, i, j}^{-1}=\frac{(-1)^{j-1} \sigma_{k, i}^{(N-j)}}{\prod_{\substack{s=1 \\ s \neq i}}^{N}\left(\rho_{k}^{(s)}-\rho_{k}^{(1)}\right)},
$$

where $\sigma_{k, i}^{(j)}=\sum_{1 \leq m_{1}<m_{2}<\ldots<m_{j} \leq N} \prod_{s=1}^{j} \rho_{k}^{\left(m_{s}\right)}\left(1-\delta_{m_{s}, i}\right)$. The matrix $H_{k+1}$ for such choice of sequences $g_{m, n, k}$ has the form

$$
H_{k+1}=\left(\begin{array}{cccc}
\rho_{k}^{(1)} & \rho_{k}^{(2)} & \ldots & \rho_{k}^{(N)} \\
\ldots & \ldots & \ldots & \ldots \\
\rho_{k}^{(1) N-1} & \rho_{k}^{(2) N-1} & \ldots & \rho_{k}^{(N) N-1} \\
\rho_{k}^{(1) N} & \rho_{k}^{(2) N} & \ldots & \rho_{k}^{(N) N}
\end{array}\right) .
$$

In the WKB approximation we suppose that all elements of the matrix $M_{k+1}^{-1} H_{k+1}$ equal zero except the diagonal ones. In this case the system of equations (51) can be rewritten as

$$
\begin{aligned}
& y_{i, k+1} \approx y_{i, k} \sum_{j=1}^{N} M_{k+1, i, j}^{-1} \rho_{k}^{(i) j}= \\
& =y_{i, k} \sum_{j=1}^{N}\left(\rho_{k}^{(i) j} \frac{(-1)^{j-1} \sigma_{k+1, i}^{(N-j)}}{\prod_{\substack{s=1 \\
s \neq i}}^{N}\left(\rho_{k+1}^{(s)}-\rho_{k+1}^{(1)}\right)}\right) .
\end{aligned}
$$

These equations are generalization to the case of the of $N$-th-order difference equation the WKB solutions obtained for the second and third-order difference equations.

\section{CONCLUSIONS}

We presented transformations of the linear difference equation into a system of the first order difference equations. The proposed transformation gives possibility to get new forms of the $N$ dimensional system of first order equations that can be useful for analysis of the solutions of the $N$-th-order difference equation. In particular, for the third-order linear difference equation 
the nonlinear second-order difference equation that plays the same role as the Riccati equation for secondorder linear equation is obtained. The new form of the $N$ dimensional system of first order equations can also be used for finding the WKB solutions of the linear difference equation with coefficients that vary sufficiently slowly with index.

\section{REFERENCES}

1. S. Elaydi. An Introduction to Difference Equations. Springer, 2005.

2. P. Cull, M. Flahive, R. Robson. Difference Equations. From Rabbits to Chaos. Springer, 2005.

3. M.I. Ayzatsky. On the matrix form of second-order linear difference equations // E-print arxiv.org/abs/ 1703.09608, 2017.

4. M.I. Ayzatsky. A note on the WKB solutions of difference equations // E-print arxiv.org/abs/1806.02196, 2018.

5. M.I. Ayzatsky. Transformation of the linear difference equation into a system of the first order difference equations // E-print arxiv.org/abs/1806.04378, 2018.
6. N.E. Tsapenko. A general method for solving a second-order homogeneous linear equation // Differ. Uravn. 1979, v. 15, № 6, p. 1050-1053.

7. V.L. Kocic, G. Ladas. Global behavior of nonlinear difference equations of higher order with applications. Kluwer, 1993.

8. Mustafa R.S. Kulenovic, G. Ladas. Dynamics of second order rational difference equations. Chapman and Hall CRC, 2001.

9. A.I. Egorov. Riccaty Equation. Pensoft Publishers, 2007.

10.P.A. Braun. WKB method for three-term recursion relations and quasienergies of an anharmonic oscillator // TMF. 1978, v. 37, № 3, p. 355-370.

11. J.S. Geronimo, D.T. Smith. WKB (Liouville-Green) Analysis od Second Order Difference Equations and Applications // Journal of Approximation Theory. 1992 , v. 69 , p. $269-301$.

12. M.H. Holmes. Introduction to perturbation methods. Springer, 2013.

13. N. Macon, A. Spitzbart. Inverses of Vandermonde Matrices // The American Mathematical Monthly. 1958 , v. 65 , № 2, p. 95-100.

Article received 28.03.2019

\section{ТРАНСФОРМАЦИЯ ЛИНЕЙНОГО РАЗНОСТНОГО УРАВНЕНИЯ В СИСТЕМУ РАЗНОСТНЫХ УРАВНЕНИЙ ПЕРВОГО ПОРЯДКА}

\section{Н.И. Айзацкий}

Представлено преобразование линейного разностного уравнения $N$-го порядка в систему разностных уравнений первого порядка. Предложенное преобразование открывает возможность получения новых форм $N$-мерной системы уравнений первого порядка, которые могут быть полезны для анализа решений разностных уравнений $N$-го порядка. В частности, для линейного разностного уравнения третьего порядка получено нелинейное разностное уравнение второго порядка, которое играет ту же роль, что и уравнение Риккати для линейного разностного уравнения второго порядка. Новая форма $N$-мерной системы уравнений первого порядка также может быть использована для нахождения ВКБ-решений линейного разностного уравнения с коэффициентами, которые медленно меняются в зависимости от индекса.

\section{ПЕРЕТВОРЕННЯ ЛІНІЙНОГО РІЗНИЦЕВОГО РІВНЯННЯ В СИСТЕМУ РІЗНИЦЕВИХ РІВНЯНЬ ПЕРШОГО ПОРЯДКУ}

\section{M.I. Айзацький}

Представлено перетворення лінійного різницевого рівняння $N$-го порядку в систему різницевих рівнянь першого порядку. Запропонована трансформація відкриває можливість отримання нових форм $N$-вимірної системи рівнянь першого порядку, які можуть бути корисними для аналізу рішень різницевих рівнянь $N$-го порядку. Зокрема, для лінійних різницевих рівнянь третього порядку отримано нелінійне різницеве рівняння другого порядку, яке відіграє ту ж саму роль, що й рівняння Ріккаті для лінійного різницевого рівняння другого порядку. Нова форма $N$-вимірної системи рівнянь першого порядку також може бути використана для пошуку ВКБ-рішень лінійного різницевого рівняння з коефіцієнтами, які повільно змінюються з індексом. 